\author{
INSTITUT FÜR INFORMATIK \\ Lehr- und Forschungseinheit für \\ Programmier- und Modellierungssprachen \\ Oettingenstraße 67, D-80538 München
}

Ludwig-

Maximilians -

Universität -

München

\title{
The XML Query Language Xcerpt: Design Principles, Examples, and Semantics
}

\author{
François Bry and Sebastian Schaffert
}

Technical Report, Computer Science Institute, Munich, Germany

http://www.pms.informatik.uni-muenchen.de/publikationen

Forschungsbericht/Research Report PMS-FB-2002-7, May 2002 


\title{
The XML Query Language Xcerpt: Design Principles, Examples, and Semantics
}

\author{
François Bry and Sebastian Schaffert \\ Institute for Computer Science, University of Munich \\ http://www.pms.informatik. uni-muenchen.de/
}

\begin{abstract}
Most query and transformation languages developed since the mid 90es for XML and semistructured data - e.g. XQuery [1], the precursors of XQuery [2], and XSLT [3] - build upon a path-oriented node selection: A node in a data item is specified in terms of a root-to-node path in the manner of the file selection languages of operating systems. Constructs inspired from the regular expression constructs $*,+$, ?, and "wildcards" give rise to a flexible node retrieval from incompletely specified data items.

This paper further introduces into Xcerpt, a query and transformation language further developing an alternative approach to querying XML and semistructured data first introduced with the language UnQL [4]. A metaphor for this approach views queries as patterns, answers as data items matching the queries. Formally, an answer to a query is defined as a simulation [5] of an instance of the query in a data item.
\end{abstract}

\section{Introduction}

Essential to semistructured data is the selection of data from incompletely specified data items. For such a data selection, a path language such as XPath [6] is convenient because it provides constructs similar to regular expressions such as *, +, ?, and "wildcards" that give rise to a flexible node retrieval. For example, the XPath expression /descendant: :a/descendant: $: \mathrm{b}$ [following-sibling: $: c$ ] selects all elements of type $b$ followed by a sibling element of type $c$ that occur at any depth within an element of type a, itself at any depth in the document.

Query and transformation languages developed since the mid 90es for XML [6] and semistructured data - e.g. XQuery [6], the precursors of XQuery, and XSLT [6] - rely upon such a path-oriented selection. They use patterns (also called templates) for expressing how the selected data, expressed by paths, are re-arranged (or re-constructed) into new data items. Thus, such languages intertwine construct parts, i.e. the construction patterns, and query parts, i.e. path selectors.

Example 1. An example for this intertwining of construct and query parts is the following XQuery query from [7]. This query creates a list of book titles for each author in a bibliography database, like that of Example 2.

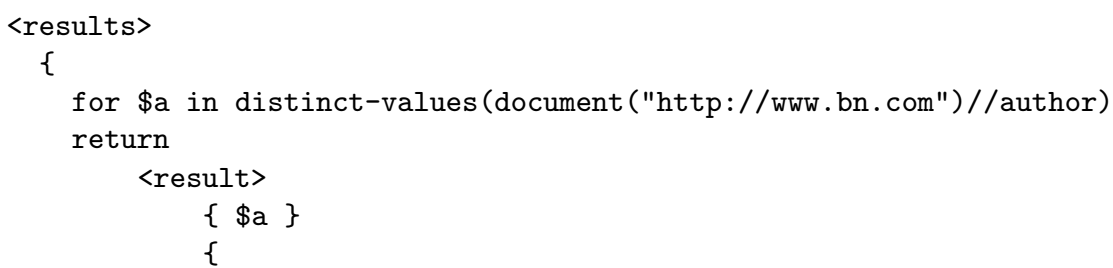




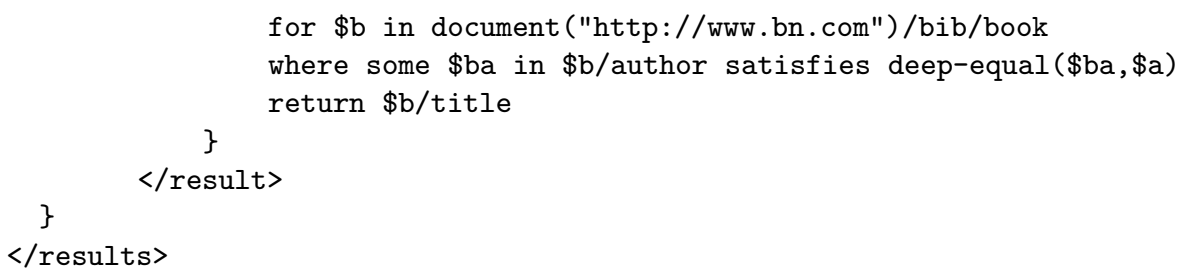

The XQuery expression is a construct pattern specifying the structure of the data to return. The query parts, i.e. the definition of the values for the variables $\$ \mathrm{a}$ and $\$ \mathrm{~b}$, are included in the construct pattern. Note that the (path-oriented) definitions of the variables $\$ \mathrm{a}$ and $\$ \mathrm{~b}$ refer to a common subpath document("http://www.bn.com"). Note also the rather complicated condition relating values of $\$ \mathrm{a}$ and $\$ \mathrm{~b}$ : some \$ba in \$b/author satisfies deep-equal (\$ba,\$a).

The same query can be expressed in Xcerpt as shown in Example 9.

This intertwining of construct and query parts à la XQuery has some advantages: For simple query-construct requests, the approach is rather natural and results in an easily understandable code. However, intertwining construct and query parts also has drawbacks:

1. Query-construct requests involving a complex data retrieval might be confusing,

2. unnecessarily complex path selections, e.g. XPath expressions involving both forward and reverse axes, are possible [8],

3. in case of several path selections, the overall structure of the retrieved data items might be difficult to grasp, as in Example 1.

Among the query and transformation languages, UnQL [4] is a noticeable exception. This language first considered using patterns instead of paths for querying semistructured data. UnQL query patterns may contain variables. Applying a kind of pattern matching algorithm, reminding of those pattern matching algorithms used in functional programming and in automated reasoning, to a UnQL query pattern and a (variable-free) data item binds the variables of the query pattern to parts of the data item. This paper further investigates this approach proposing the following new ideas:

1. Instead of pattern matching, a (non-standard form of) unification is considered using which two query patterns, both containing variables, can be made identical through bindings of their variables.

2. Within a query pattern, a variable might be constrained through a (sub-)pattern to be bound only to data conforming to this (sub-)pattern.

3. Instead of building upon the functional paradigm, as UnQL does, the paradigm of SQL and of logic programming is retained. Thus, a query might have several answers and the choice of some or all of the answers specified by a query can be expressed with language constructs reminding of the well-known set operators of elementary mathematics.

4. A chaining of queries, the answers to which are not necessarily sought for, makes it possible to rather naturally split complex queries into intuitive parts. 
A metaphor for this approach is to see queries as forms, answers as form fillings yielding database items. With this approach, patterns are used not only in construct expressions, but also for data selection.

In the following, the basic concepts of a query language called Xcerpt are introduced. An answer to a query in this language is formalized as a simulation [5] of a ground instance of the query in a database item. This formalization yields a compositional semantics.

\section{Language Principles}

The following principles have prevailed to the definition of the query language:

Pattern-based or positional instead of navigational. A query should correspond to a form, an answer to a filling yielding a database item. The relative positions of variables in a query should be easily recognizable. It should also be possible to constrain a variable in a query, within this query to some pattern.

Referential transparency. The meaning of an expression, especially of a variable, should be the same wherever it appears. Therefore, destructive assignments are prohibited and variables must be functional or logic programming variables.

Compositional semantics. A (structurally) recursive definition of the semantics of a query in terms of the semantics of its parts, i.e. a Tarski-style model theory, is sought for.

Multiple variable bindings. Like with SQL and other query languages, queries might have several answers, each answer binding the query variables differently.

Strict separation of construct and query proper. Query expressions should not occur in construct patterns. In construct expressions only variables should occur, not conditions on the variables. Conditions on variables should occur only in query expressions.

Symmetry. Queries should allow similar forms of incomplete specifications in breadth, i.e. concerning siblings, and in depth, i.e. concerning children.

Circularity. For the query language, queries and answers should be a queryable data items. Note that this is more stringent than requiring an XML representation of queries: E.g. the existence of JavaML does not make XML a data type directly accessible in Java.

Note that the requirements of [9] are fulfilled by or compatible with the basic query language defined below.

\section{Xcerpt Basic Constructs}

This section introduces the essential constructs of the query language Xcerpt. Aspects of XML, such as attributes and namespaces, that are irrelevant to this paper, are not explicitly addresses in the following.

Below, the following pairwise disjoint sets of symbols are referred to: A set $\mathcal{I}$ of identifiers, a set $\mathcal{L}$ of labels (or tags or strings), a set $\mathcal{V}_{l}$ of label variables, 
a set $\mathcal{V}_{t}$ of term (or data item) variables. Identifiers are denoted by id, labels (variables, resp.) by lower (upper, resp.) case letters with or without indices. The following meta-variables (with or without indices and/or superscripts) are used: $i d$ denotes an identifier, $l$ denotes a label, $L$ a label variable, $X$ a term variable, $t$ a term (as defined below), $v$ a label or a term, and $V$ a label or term variable.

\subsection{Database Terms}

A database is a set (or multiset) of database terms. The children of a document node may be either ordered (as in standard XML), or unordered. In the following, a term whose root is labelled $l$ and has ordered (unordered, resp.) children $t_{1}, \ldots, t_{n}$ is denoted $l\left[t_{1}, \ldots, t_{n}\right]\left(l\left\{t_{1}, \ldots, t_{n}\right\}\right.$, resp. $)$.

Definition 1 (Database Terms). Xcerpt Database Terms are expressions inductively defined as follows and satisfying Conditions 1 and 2 given below:

1. If $l$ is a label, then $l$ is a (atomic) database term.

2. If id is an identifier and $t$ is a database term neither of the form $i d_{0}: t_{0}$ nor of the form $\uparrow i d_{0}$, then $i d: t$ is a database term.

3. If id is an identifier, then $\uparrow i d$ is a database term.

4. If $l$ is a label and $t_{1}, \ldots, t_{n}$ are $n \geq 1$ database terms, then $l\left[t_{1}, \ldots, t_{n}\right]$ and $l\left\{t_{1}, \ldots, t_{n}\right\}$ are database terms.

Condition 1: For a given identifier id an identifier definition $i d$ : $t_{0}$ occurs at most once in a term.

Condition 2: For every identifier reference $\uparrow i d$ occurring in a term $t$ an identifier definition id: $t_{0}$ occurs in $t$.

Example 2. The following Xcerpt database terms describe the book offers of two online book stores bn.com and amazon.com (This example is inspired from the W3C XQuery Use-Cases [7]). Note that both bookstores rely on different data formats.

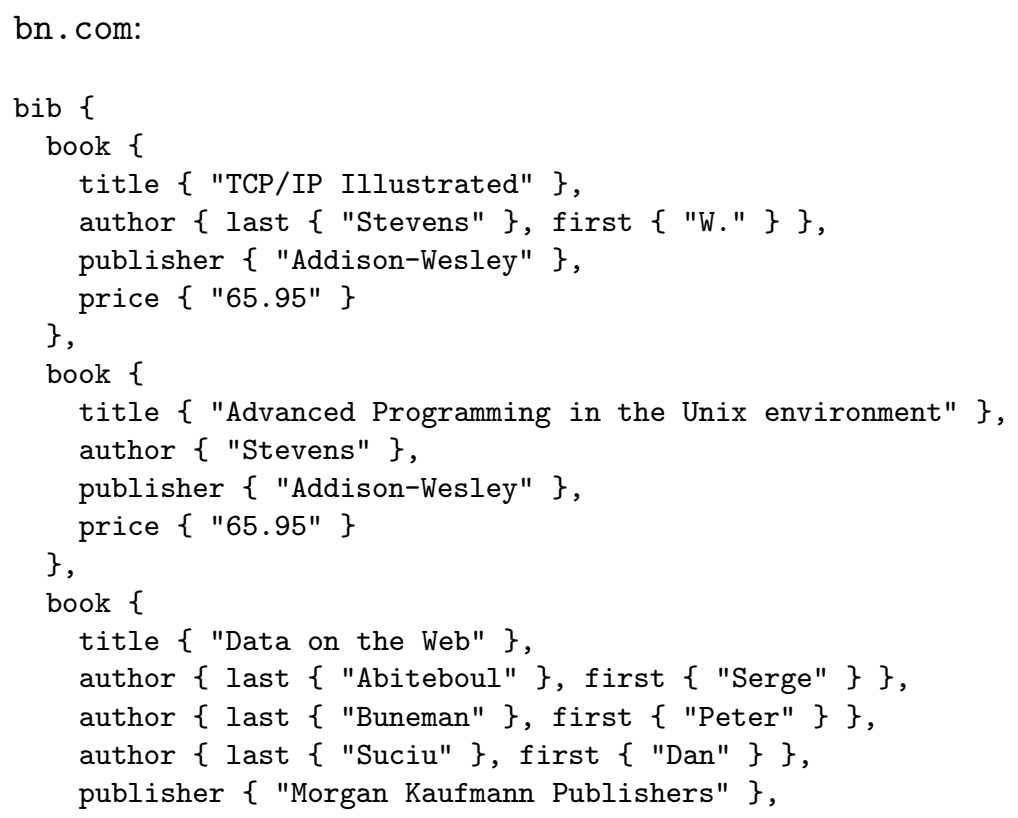




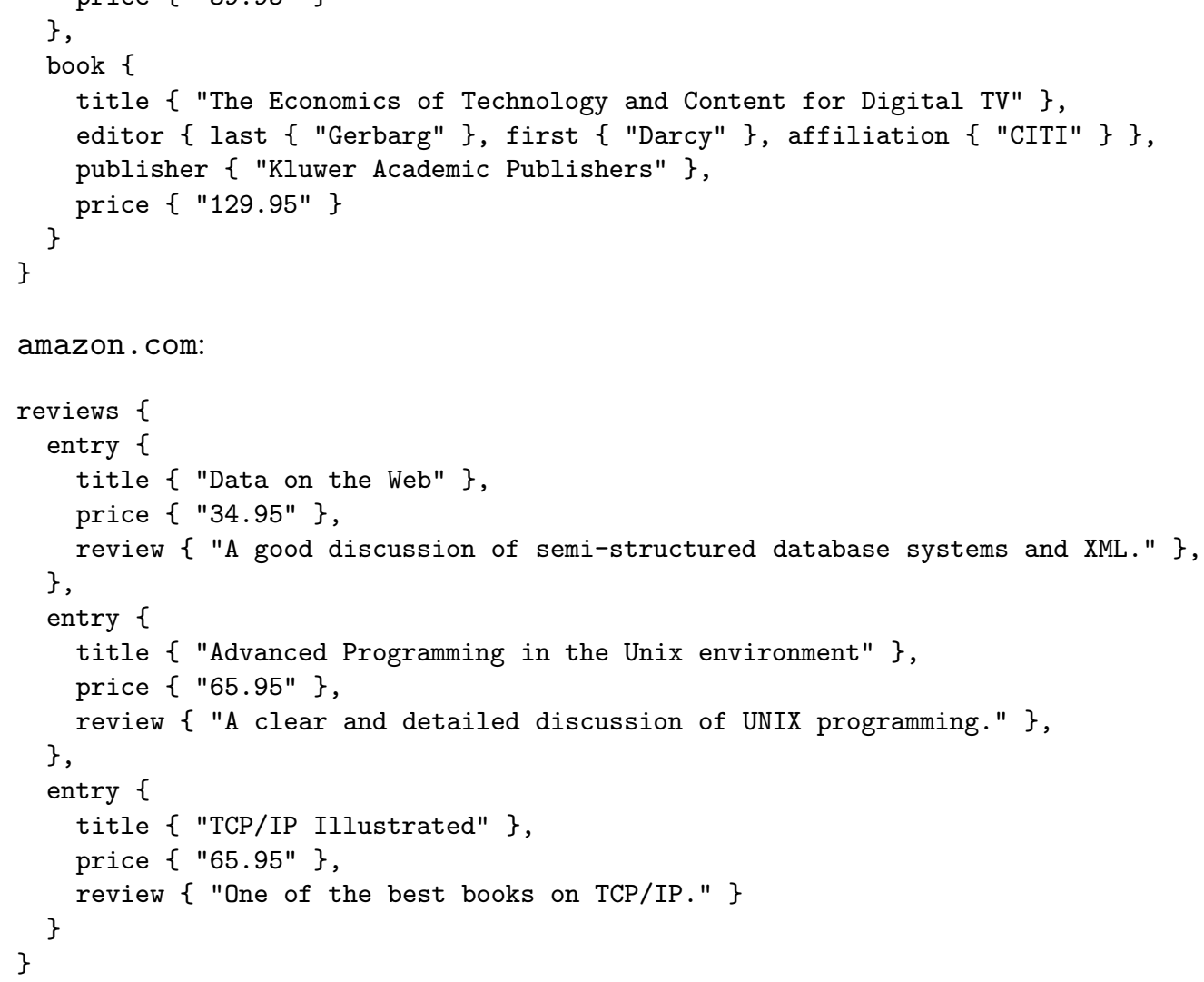

Note that in both examples the element order is of no importance. This is expressed in the Xcerpt syntax using the single curly brackets \{\} .

\subsection{Query Terms}

A query term is a pattern that specifies a selection of database terms very much like logical atoms and SQL selections do. The evaluation of query terms (cf. below Definition 12 for a formalisation) differs from the evaluation of logical atoms and SQL selections as follows:

1. Answers might have additional subterms to those mentioned in the query term.

2. Answers might have another subterm ordering than the query.

3. A query term might specify subterms at an unspecified depth.

In query terms, the single square and curly brackets, [ ] and \{\} , denote "exact subterm patterns", i.e. single (square or curly) brackets are used in a query term to be answered by database terms with no more subterms than those given in the query term. Double square and curly brackets, [[]] and $\{\{\}\}$, on the other hand, denote "partial subterm patterns".

[ ] and [[ ]] are used if the subterm order in the answers is to be that of the query term, \{\} and $\{\{\}\}$ are used otherwise. Thus, possible answers to the query term $t_{1}=a[b, c\{\{d, e\}\}, f]$ are the database terms $a[b, c\{d, e, g\}, f]$ and $a[b, c\{d, e, g\}, f\{g, h\}]$ and $a[b, c\{d, e\{g, h\}, g\}, f\{g, h\}]$ and $a[b, c[d, e], f]$. In contrast, $a[b, c\{d, e\}, f, g]$ and $a\{b, c\{d, e\}, f\}$ are no answers to $t_{1}$. The only answers to $f\{\}$ are f-labelled database terms with no children. 
In a query term, a term variable $X$ can be constrained to some query terms using the construct $\leadsto$, read "as". Thus, the query term $t_{2}=a\left[X_{1} \leadsto\right.$ $\left.b[c, d], X_{2}, e\right]$ constrains the term variable $X_{1}$ to such database terms that are possible answers to the query term $b[c, d]$. Note that the term variable $X_{2}$ is unconstrained in $t_{2}$. Possible answers to $t_{2}$ are $a[b[c, d], f, e]$ which binds $X_{1}$ to $b[c, d]$ and $X_{2}$ to $f, a[b[c, d], f[g, h], e]$ which binds $X_{1}$ to $b[c, d]$ and $X_{2}$ to $f[g, h]$, $a[b[c, d, e], f, e]$ which binds $X_{1}$ to $b[c, d, e]$ and $X_{2}$ to $f$, and $a[b[c, e, d], f, e]$ which binds $X_{1}$ to $b[c, e, d]$ and $X_{2}$ to $f$. In query terms, the construct desc, read "descendant", specifies a subterm at an unspecified depth. Thus, possible answers to the query term $t_{3}=a[X \leadsto \operatorname{desc} f[c, d], b]$ are $a[f[c, d], b]$ and $a[g[f[c, d]], b]$ and $a[g[f[c, d], h], b]$ and $a[g[g[f[c, d]]], b]$ and $a[g[g[f[c, d], h], i], b]$.

Definition 2 (Query Terms). Xcerpt Query terms are expressions inductively defined as follows and satisfying Conditions 1 and 2 of Definition 1:

1. If $l$ is a label and $L$ is a label variable, then $l, L, l\{\{\}\}$, and $L\{\{\}\}$ are (atomic) query terms.

2. A term variable is a query term.

3. If $i d$ is an identifier and $t$ is a query term neither of the form $i d_{0}: t_{0}$ nor of the form $\uparrow i d_{0}$, then $i d: t$ is a query term.

4. If id is an identifier, then $\uparrow$ id is a query term.

5. If $X$ is a variable and $t$ a query term, then $X \leadsto t$ is a query term.

6. If $X$ is a variable and $t$ is a query term, then $X \leadsto$ desc $t$ is a query term.

7. If $l$ is a label, $L$ a label variable and $t_{1}, \ldots, t_{n}$ are $n \geq 1$ query terms, then $l\left[t_{1}, \ldots, t_{n}\right], L\left[t_{1}, \ldots, t_{n}\right], l\left\{t_{1}, \ldots, t_{n}\right\}, L\left\{t_{1}, \ldots, t_{n}\right\}, l\left[\left[t_{1}, \ldots, t_{n}\right]\right]$, $L\left[\left[t_{1}, \ldots, t_{n}\right]\right], l\left\{\left\{t_{1}, \ldots, t_{n}\right\}\right\}$, and $L\left\{\left\{t_{1}, \ldots, t_{n}\right\}\right\}$ are query terms.

Query terms in which no variables occur are ground. Query terms that are not of the form $\uparrow i d$, are strict. The leftmost label of strict and ground query terms of the form $l, l\{\{\}\}, l\left\{t_{1}, \ldots, t_{n}\right\}$, and $l\left[t_{1}, \ldots, t_{n}\right]$ is $l$; the leftmost label of a strict and ground query term of the form id: $t$ is the leftmost label of $t$.

Note that desc never occurs in a ground query term, for it is by Definition 2 always coupled with a variable.

Example 3. Consider the bookstore databases of Example 2. The following simple query term could query the first database for titles and authors and bind the variables TITLE and AUTHOR to the corresponding values in bn.com's database:

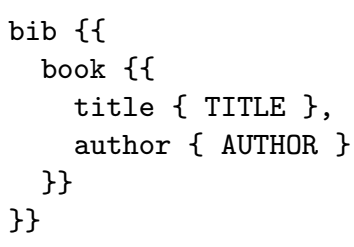

The evaluation strategy of Xcerpt is based on so-called Simulation Unification. It is explained in more detail in [11]. The two variables TITLE and AUTHOR will have several possible bindings as a result of the simulation unification, representing all valid combinations of a title with an author that can be found in the database, e.g. AUTHOR="Dan Suciu" and TITLE="Data on the Web" or AUTHOR="Serge Abiteboul" and TITLE="Data on the Web". 
Example 3 would bind the variables to the "leafs" of the database terms. Xcerpt also allows variables at a "higher position" in a query term, as illustrated in the next example.

Example 4. The following Xcerpt query binds the variable TITLE to the compound element title $\{\ldots\}$ (thus retrieving not the "leaf" but the parent element title):

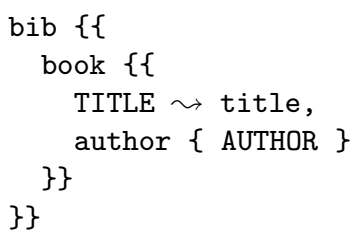

Thanks to Simulation Unification (cf. below Section 4), the "leaf" of a title element does not have to be explicitly mentioned in the query of Example 4 for being included in the answers.

Finally, the descendant construct serves to express indefiniteness.

Example 5. The following Xcerpt query retrieves the titles of books with an author "Stevens" at any depth:

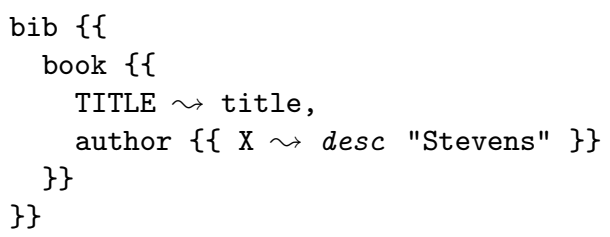

Definition 2 requires a desc expression to be preceded by $X \leadsto$ for some variable $X$. This is convenient for simplifying the formalisation of Xcerpt's declarative semantics (cf. below Definition 10). However, this is dispensible in practice (at the cost of a more complicated counterpart in Definition 10).

Example 6. Example 5 can be expressed using the following query term (although not conforming to Definition 2):

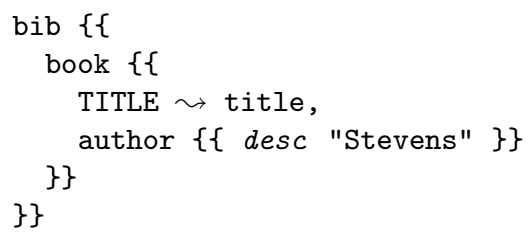

In a query term, multiple occurrences of a same term variable are not precluded. E.g. a possible answer to $a\{\{X \leadsto b\{\{c\}\}, X \leadsto b\{\{d\}\}\}$ is $a\{b\{c, d\}\}$. However, $a[[X \leadsto b\{c\}, X \leadsto f\{d\}]]$ has no answers, for labels $b$ and $f$ are distinct.

Child subterms and subterms of query terms are defined such that if $t=f[a, g\{Y \leadsto \operatorname{desc} b\{X\}, h\{a, X \leadsto k\{c\}\}]$, then $a$ and $g\{Y \leadsto$ desc $b\{X\}, h\{a, X \leadsto k\{c\}\}$ are the only child subterms of $t$ and e.g. $a$ and 
$X$ and $Y \leadsto \operatorname{desc} b\{X\}$ and $h\{a, X \leadsto k\{c\}\}$ and $X \leadsto k\{c\}$ and $t$ itself are subterms of $t$. Note that $f$ is not a subterm of $t$.

The $\leadsto$ construct makes it possible to express (undesirable) "cyclic" query terms. Definition 3 avoids such "cyclic" query terms.

Definition 3 (Variable Well-Formed Query Terms). A term variable $X$ depends on a term variable $Y$ in a query term $t$ if $X \leadsto t_{1}$ is a subterm of $t$ and $Y$ is a subterm of $t_{1}$. A query term $t$ is variable well-formed if $t$ contains no term variables $X_{0}, \ldots, X_{n}(n \geq 1)$ such that 1. $X_{0}=X_{n}$ and 2. for all $i=1, \ldots, n, X_{i}$ depends on $X_{i-1}$ in $t$.

E.g. $f\{X \leadsto g\{X\}\}$ and $f\{X \leadsto g\{Y\}, Y \leadsto h\{X\}\}$ are not variable wellformed. Variable well-formedness precludes queries specifying infinite answers. In the following, query terms are assumed to be variable well-formed.

\subsection{Construct Terms}

Xcerpt Construct terms serve to re-assemble variables, the "values" of which are specified in query terms, so as to form new database terms. Thus, like in database terms both constructs [] and \{\} can occur in construct terms. Variables as references to subterms specified in a query can also occur in construct terms. However, the construct $\leadsto$ is not allowed in construct terms. The rationale for forbidding $\leadsto$ in construct terms is that variables should be constrained where they are defined, i.e. in query terms, not in construct terms where they are used to specify new terms.

Since querying a database may yield multiple alternative bindings for the same variables, it might be desirable to collect all such bindings in the construction of a result. The construct all serves this purpose. all $t$ denotes the collection of all instances of $t$ (binding the variables free in term $t$ in all possible ways and recursively evaluating nested all constructs). A variable $X$ is free in $t$, if $X$ is not already contained within the argument of an all construct.

Definition 4 (Construct Terms). Xcerpt Construct terms are expressions inductively defined as follows satisfying Conditions 1 and 2 of Definition 1:

1. Labels and label variables are (atomic) construct term.

2. If id is an identifier and $t$ is a construct term, then id: $t$ is a construct term.

3. If id is an identifier, then $\uparrow i d$ is a construct term.

4. A term variable is a construct term.

5. If $t$ is a construct term, then all $t$ is a construct term.

6. If $l$ is a label, $L$ is a label variable and $t_{1}, \ldots, t_{n}$ are $n \geq 1$ construct terms, then $l\left[t_{1}, \ldots, t_{n}\right], L\left[t_{1}, \ldots, t_{n}\right], l\left\{t_{1}, \ldots, t_{n}\right\}$, and $L\left\{t_{1}, \ldots, t_{n}\right\}$ are construct terms.

Note that construct terms that are not of the form all $t$ are (simple kinds of) query terms and database terms are (simple kinds of) construct terms.

Example 7. Consider the database bn.com of Example 2. Assume that some query term (e.g. that of Example 3) binds the variables TITLE and AUTHOR. The construct term 


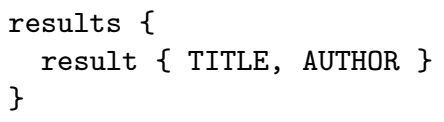

assembles the bindings of TITLE and AUTHOR into new database terms like e.g.

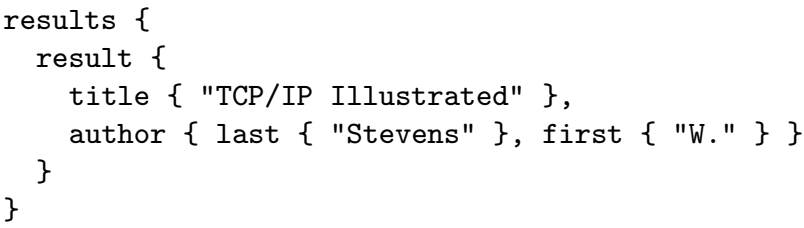

In the general case there are several alternative bindings for the variables TITLE and AUTHOR, e.g. several values for TITLE. The all construct may be used to collect all such alternatives:

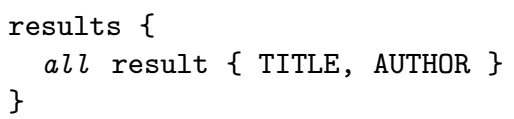

This yields as a result an unordered collection of result elements, collecting all possible combinations for TITLE and AUTHOR, e.g. like in

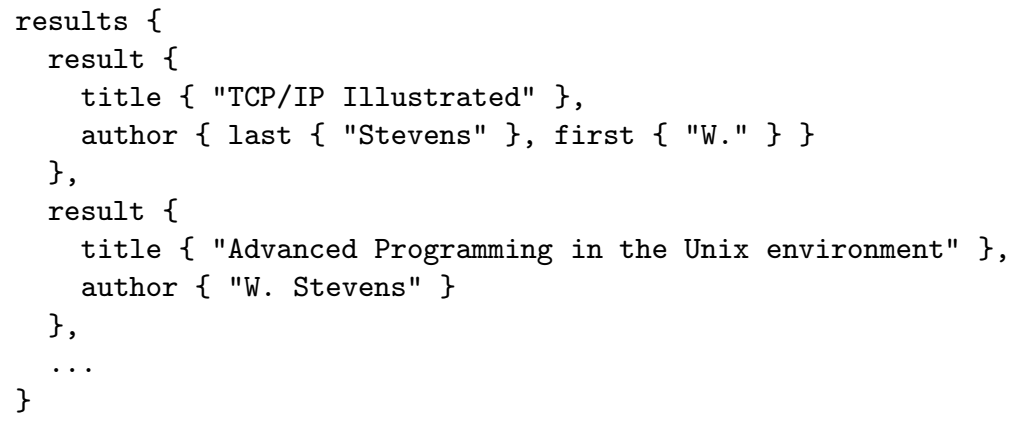

Example 8. Using the all construct, the XQuery expression of Example 1 returning for each author a list of all his titles can be expressed in Xcerpt as follows:

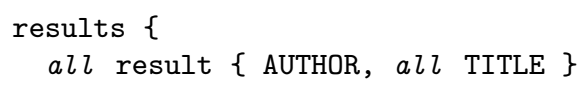

The symmetric query listing for each title all its authors is expressed in Xcerpt as follows:

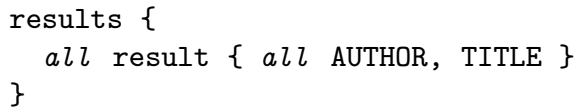

Note that the only change from the first to the second Xcerpt construct term is the position of the all construct. The same query from Example 3 can be used in both cases, as opposed to XQuery which requires two completely different queries (cf. queries Q3 and Q4 of use case "XMP" in [7]). 


\subsection{Construct-Query Rules}

Xcerpt Construct-query rules relate queries, consisting of a conjunction of query terms, and construct terms. It is assumed (cf. below Point 3 of Definition 5) that each term variable occurring (left or right of $\leadsto$ or elsewhere) in the construct term of a construct-query rule also occurs in at least one of the query terms of the rule, i.e. variables in construct-query rules are assumed to be "rangerestricted" or "allowed".

Definition 5 (Construct-Query Rule). A construct-query rule is an expression of the form $t^{c} \leftarrow t_{1}^{q} \wedge \ldots \wedge t_{n}^{q}$ such that:

1. $n \geq 1$ and for all $i=1, \ldots n, t_{i}^{q}$ is a query term,

2. $t^{c}$ is a construct term, and

3. every variable occurring in $t^{c}$ also occurs in at least one of the $t_{i}^{q}$.

The left hand-side, i.e. the construct term, of a (construct-query) rule will be referred to as the rule "head". The right hand-side of a (construct-query) rule will be referred to as the rule "body". Note that, in contrast to the body of a Prolog clause, the body of a (construct-query) rule cannot be empty, for empty rule bodies do not seem to be needed for the applications considered.

Example 9. The following construct-query rule combines the query and construct terms used in the previous Examples 3 and 8:

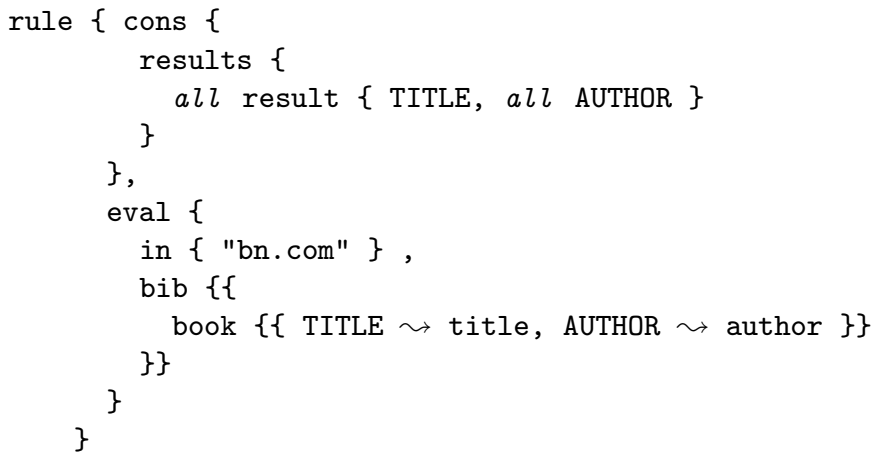

The advantage of the clear separation between construct and query parts in Xcerpt is obvious, if you recall Example 1. The rule in Example 9 also demonstrates the circularity of Xcerpt: a rule is itself a term.

Note that the eval part contains an in construct. This construct allows to specify a different resource for each query term. The rationale behind this is illustrated on the following, more complex example.

Example 10. The following rule creates a list of books with their prices in both stores bn.com and amazon.com:

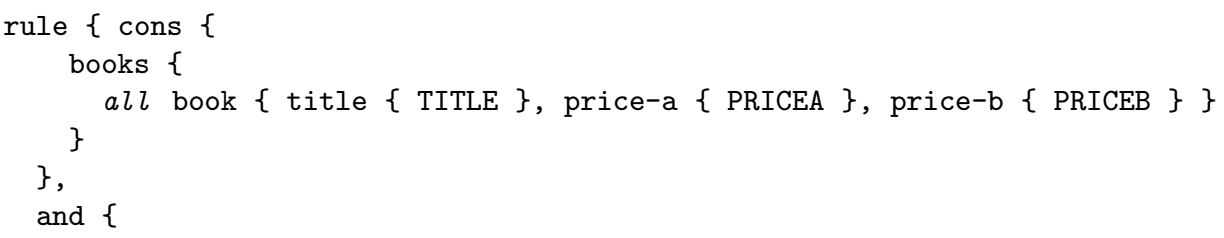




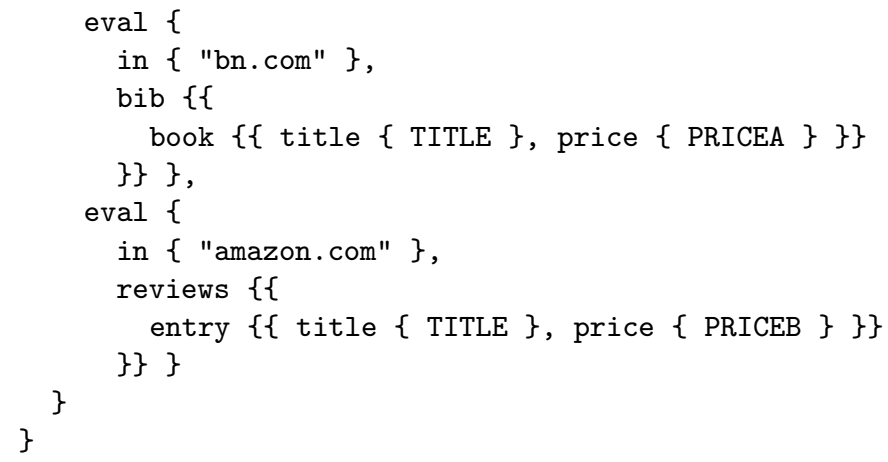

Note is that the combination of query terms in example 10 expresses an equijoin on the book title.

\subsection{Xcerpt Programs}

An Xcerpt program consists of one or several construct-query rules and of a "main query". A notion of modules (cf. below Section 3.7) makes it possible to combine and re-use parts of Xcerpt programs in different manners.

\subsection{Rule Chaining}

Xcerpt allows to "chain" rules, i.e. to evaluate one rule against the result of another rule. This allows for very complex queries and transformations, encapsulating subqueries and calculations in separate rules.

Example 11. Consider the rule of Example 10. Assume the data constructed is to be further transformed into two different formats, HTML [12] and WML [13], the one suitable for a PC screen, the other suitable for the small screen of a PDA (personal digital assistand). In Xcerpt, this could be expressed using additional rules that query the "result" of the first rule. A transformation into an HTML table and WML card could look like this:

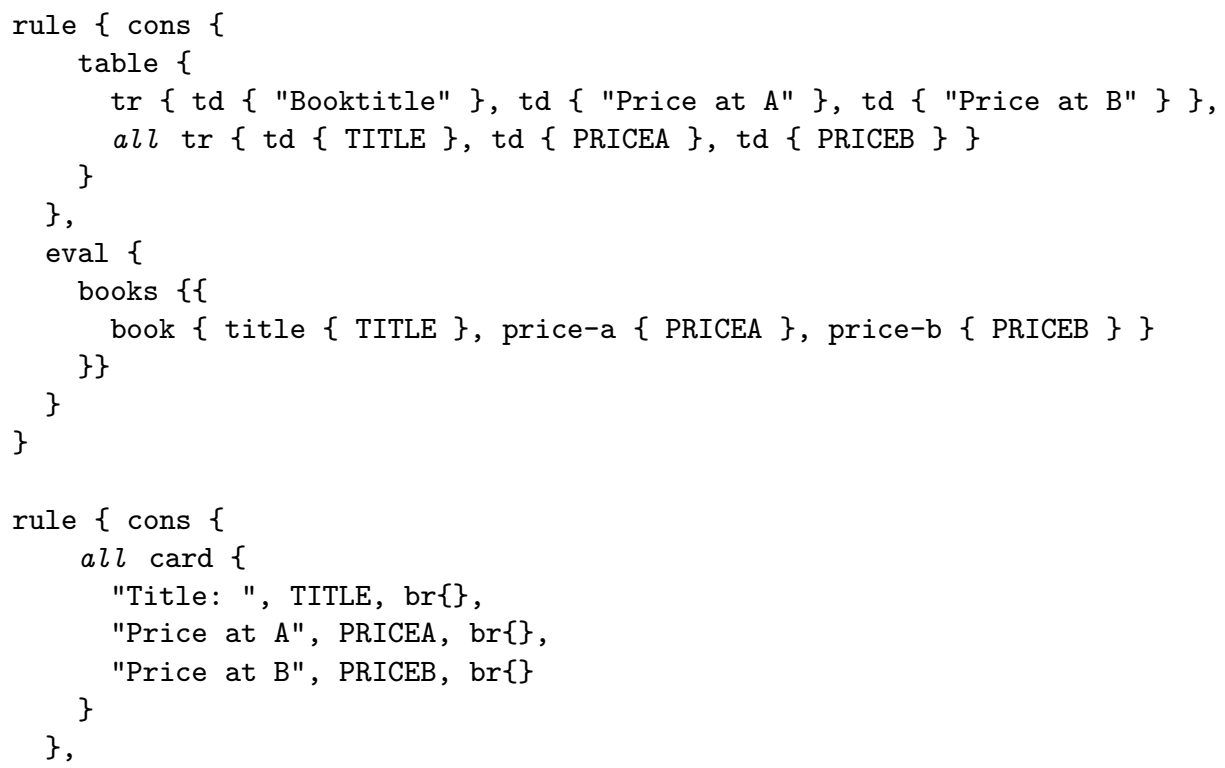




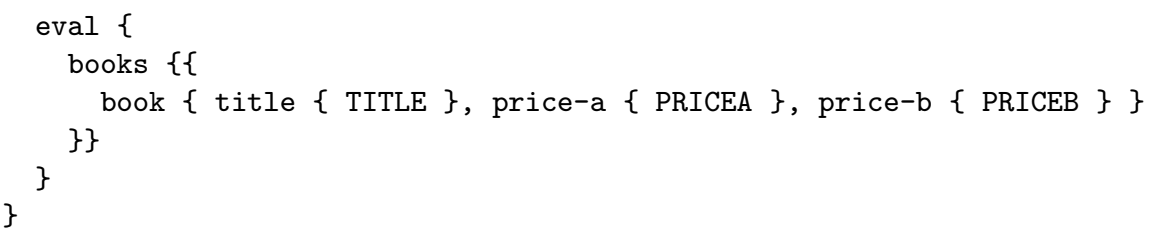

Both a forward chaining (as in deductive databases) and a backward chaining (as in Prolog) are possible and reasonable for processing Xcerpt rules. Backward chaining can be very efficient but requires a "unification" of query and construct terms. Xcerpt relies on a non-standard unification called Simulation Unification. Simulation Unification is introduced below in Section 4.

\subsection{Further Language Constructs}

The previous sections describe the basic constructs of the language Xcerpt. While these are sufficient for basic queries and transformations, a query language also needs to provide higher-level constructs, e.g. arithmetics and aggregations. This section gives a short overview over additional features of Xcerpt currently under development. This list is non-comprehensive.

Basic datatypes. In this article, only string data are considered, although Xcerpt's support of various basic scalar types such as different kinds of numbers (e.g. integers and reals) is under development. The (current) view is that Xcerpt will support the "simple types" of XML Schema $[14,15,16]$ including basics operations on these types (such as e.g. basic arithmetics on number types).

Elementary text processing primitives. In addition to the primitives forseen in XML Schema $[14,15,16]$ for the simple types "string", "normalizedString", and "token", Xcerpt includes primitives (inspired from Perl [17]) for an an elementary text processing - among others, regular expressions for text selection.

Aggregation. In re-assembling answers to queries into new data items, one often needs to collect several answers (as with the all construct, cf. Section 3.3) or to compute values (such as an average) from collected answers. In addition to the all construct, Xcerpt supports standard aggregation primitives such as avg (average), max, min for number datatypes, and concat (concatenation) for text datatype. The some construct gives rise to a non-deterministic selection of one answer. This construct is a declarative counterpart to Prolog's cut (!) reminding of Prolog's once.

User defined constraints. Xcerpt allows the user to specify additional constraints to variables occuring in query terms. These user defined constraints may be expressed in terms of simulation unification (cf. below Section 4) or using system or user-defined functions.

system and user-defined functions. It is possible to refer in an Xcerpt program to functions specified (e.g. by the user) outside the Xcerpt program. 
Polymorphic Type system. A type system has two advantages: Programming errors can be detected at compile time (thus supporting program development) and the processing of queries can be more efficient. A extensible polymorphic type system à la ML [18] for Xcerpt is under development using which user defined types are expressed in an XML Schema syle $[14,15,16]$.

Declarations and shadowing. Variable and type declarations local to part of an Xcerpt program make it possible that some definitions and names are local to a program part. Shadowing makes it possible to to differently binds same names within different program parts.

Modules. Modules aqrte under developments using which parts of Xcerpt programs can be imported and exported so as to combine parts of programs in different manners and to hide parts of programs that have no global relevance.

\section{Query Semantics}

Xcerpt's query semantics is based on graph simulation. Informally, a simulation of a graph $G_{1}$ in a graph $G_{2}$ is a mapping of the nodes of $G_{1}$ in the nodes of $G_{2}$ preserving the edges. The graphs considered are directed, ordered and rooted and their nodes are labelled.

Definition 6 (Graph Simulation). Let $G_{1}=\left(V_{1}, E_{1}\right)$ and $G_{2}=\left(V_{2}, E_{2}\right)$ be two graphs and let $\sim$ be an equivalence relation on $V_{1} \cup V_{2}$. A relation $\mathcal{S} \subseteq V_{1} \times V_{2}$ is a simulation with respect to $\sim$ of $G_{1}$ in $G_{2}$ if:

1. If $v_{1} \mathcal{S} v_{2}$, then $v_{1} \sim v_{2}$.

2. If $v_{1} \mathcal{S} v_{2}$ and $\left(v_{1}, v_{1}^{\prime}\right) \in E_{1}$, then there exists $v_{2}^{\prime} \in V_{2}$ such that $v_{1}^{\prime} \mathcal{S} v_{2}^{\prime}$ and $\left(v_{2}, v_{2}^{\prime}\right) \in E_{2}$.

A simulation $\mathcal{S}$ of a tree $T_{1}$ with root $r_{1}$ in a tree $T_{2}$ with root $r_{2}$ is a rooted simulation of $T_{1}$ in $T_{2}$ if $r_{1} \mathcal{S} r_{2}$.

Definition 7 (Graphs Induced by Strict and Ground Query Terms). Let $t$ be a strict and ground query term. The graph $G_{t}=\left(N_{t}, V_{t}\right)$ induced by $t$ is defined by:

1. $N_{t}$ is the set of strict subterms (cf. Definition 2) of $t$ and each $t^{\prime} \in N_{t}$ is labelled with the leftmost label (cf. Definition 2) of $t^{\prime}$.

2. $V_{t}$ is the set of pairs $\left(t_{1}, t_{2}\right)$ such that either $t_{2}$ is a child subterm of $t_{1}$, or $\uparrow i d$ is a child subterm of $t_{1}$ and the identifier definition id: $t_{2}$ occurs in $t$.

3. The children of a node are ordered in $G_{t}$ like in $t$.

Note that $t$ is the root of $G_{t}$.

Figure 1 illustrates Definition 7 . Note that the graph induced by a ground query term as defined in Definition 7 does not fully convey the term structure: Missing are representations of the various nestings [ ], \{\} , [[ ]] and $\{\{\}\}$.

Below, a database term is identified with the graph it induces.

Definition 8 (Ground Query Term Simulation). Let $t_{1}$ and $t_{2}$ be ground query terms. Let $S_{i}$ denote the set of subtrees of $t_{i}(i \in\{1,2\})$. A relation $\mathcal{S} \subseteq S_{1} \times S_{2}$ is a ground query term simulation of $t_{1}$ in $t_{2}$ if: 


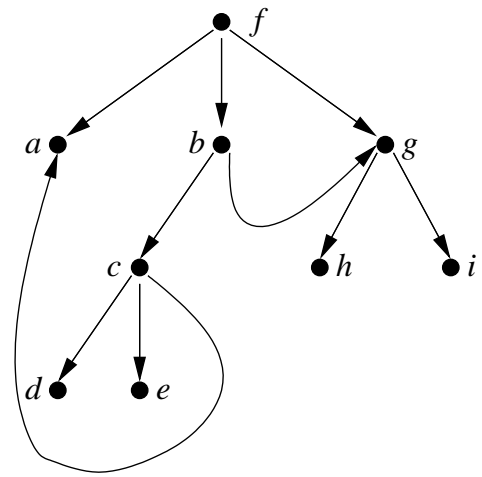

(a) Abstract node representation

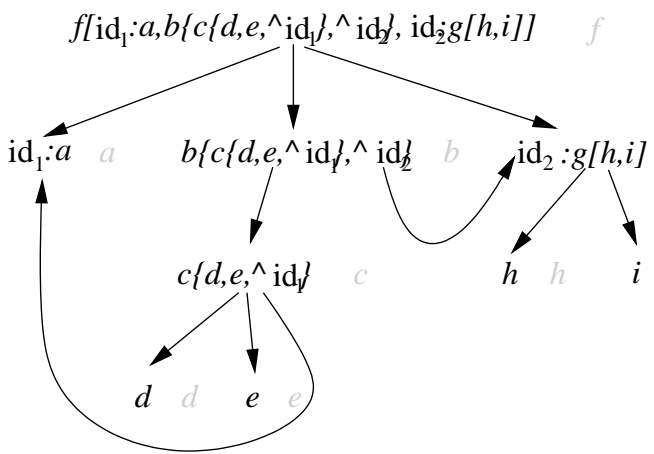

(b) Full node representation (node labels in gray)

Fig. 1. Graph induced by $t=f\left[\operatorname{id}_{1}: a, b\left\{c\left\{d, e, \uparrow \operatorname{id}_{1}\right\}, \uparrow \operatorname{id}_{2}\right\}, \operatorname{id}_{2}: g[h, i]\right]$.

1. $t_{1} \mathcal{S} t_{2}$.

2. If $l_{1} \mathcal{S} l_{2}$, then $l_{1}=l_{2}$.

3. If $\left.l_{1}\left\{\left\{t_{1}^{1}, \ldots, t_{n}^{1}\right\}\right\} \mathcal{S} l_{2}\left\{\left\{t_{1}^{2}, \ldots, t_{m}^{2}\right\}\right\}\right)$, then $l_{1}=l_{2}$ and for all $i \in\{1, \ldots, n\}$ there exists $j \in\{1, \ldots, m\}$ such that $t_{i}^{1} \mathcal{S} t_{j}^{2}$.

4. If $\left.l_{1}\left\{\left\{t_{1}^{1}, \ldots, t_{n}^{1}\right\}\right\} \mathcal{S} l_{2}\left\{t_{1}^{2}, \ldots, t_{m}^{2}\right\}\right)$, then $l_{1}=l_{2}$ and for all $i \in\{1, \ldots, n\}$ there exists $j \in\{1, \ldots, m\}$ such that $t_{i}^{1} \mathcal{S} t_{j}^{2}$ ).

5. If $\left.l_{1}\left\{t_{1}^{1}, \ldots, t_{n}^{1}\right\} \mathcal{S} l_{2}\left\{\left\{t_{1}^{2}, \ldots, t_{m}^{2}\right\}\right\}\right)$, then $l_{1}=l_{2}$ and for all $i \in\{1, \ldots, n\}$ there exists $j \in\{1, \ldots, m\}$ such that $t_{i}^{1} \mathcal{S} t_{j}^{2}$, and for all $j \in\{1, \ldots, m\}$ there exists $i\{1, \ldots, n\}$ such that $t_{i}^{1} \mathcal{S} t_{j}^{2}$.

6. If $\left.l_{1}\left\{t_{1}^{1}, \ldots, t_{n}^{1}\right\} \mathcal{S} l_{2}\left\{t_{1}^{2}, \ldots, t_{m}^{2}\right\}\right)$, then $l_{1}=l_{2}$ and for all $i \in\{1, \ldots, n\}$ there exists $j \in\{1, \ldots, m\}$ such that $t_{i}^{1} \mathcal{S} t_{j}^{2}$, and for all $j \in\{1, \ldots, m\}$ there exists $i\{1, \ldots, n\}$ such that $t_{i}^{1} \mathcal{S} t_{j}^{2}$.

Definition 9 (Simulation Preorder). $\preceq$ is the preorder on the set of ground query terms defined by $t_{1} \preceq t_{2}$ if there exists a ground query term simulation of $t_{1}$ in $t_{2}$.

Figure 2 illustrates Definition 8. The simulation of Figure 2 is minimal for $\subseteq$ in the sense that no strict subset of this simulation relation is a simulation of $t^{q}$ in $t^{d b}$.

By Definition 8, label identity is a rooted simulation of every ground query term in itself. By Definition 8, if $\mathcal{S}_{1}$ is a ground query term simulation of $t_{1}$ in $t_{2}$ and if $\mathcal{S}_{2}$ is a ground query term simulation of $t_{2}$ in $t_{3}$, then $\mathcal{S}=\left\{\left(l_{1}, l_{3}\right) \mid\right.$ $\left.\exists l_{2}\left(l_{1}, l_{2}\right) \in \mathcal{S}_{1} \wedge\left(l_{2}, l_{3}\right) \in \mathcal{S}_{2}\right\}$ is a ground query term simulation of $t_{1}$ in $t_{3}$. In other word, $\preceq$ is reflexive and transitive, i.e. it is a preorder on the set of database terms.

However, $\preceq$ is not a partial order, for although $t_{1}=f\{a\} \preceq t_{2}=f\{a, a\}$ and $t_{2}=f\{a, a\} \preceq t_{1}=f\{a\}$ (both $a$ of $t_{2}$ can be simulated by the same $a$ of $\left.t_{1}\right), t_{1}=f\{a\} \neq t_{2}=f\{a, a\}$.

Rooted simulation with respect to label equality is a first notion towards a formalisation of answers to query terms: If there exists a ground query term simulation of a ground query term $t_{1}$, in a database term $t_{2}$, then $t_{2}$ is an answer to $t_{1}$. 


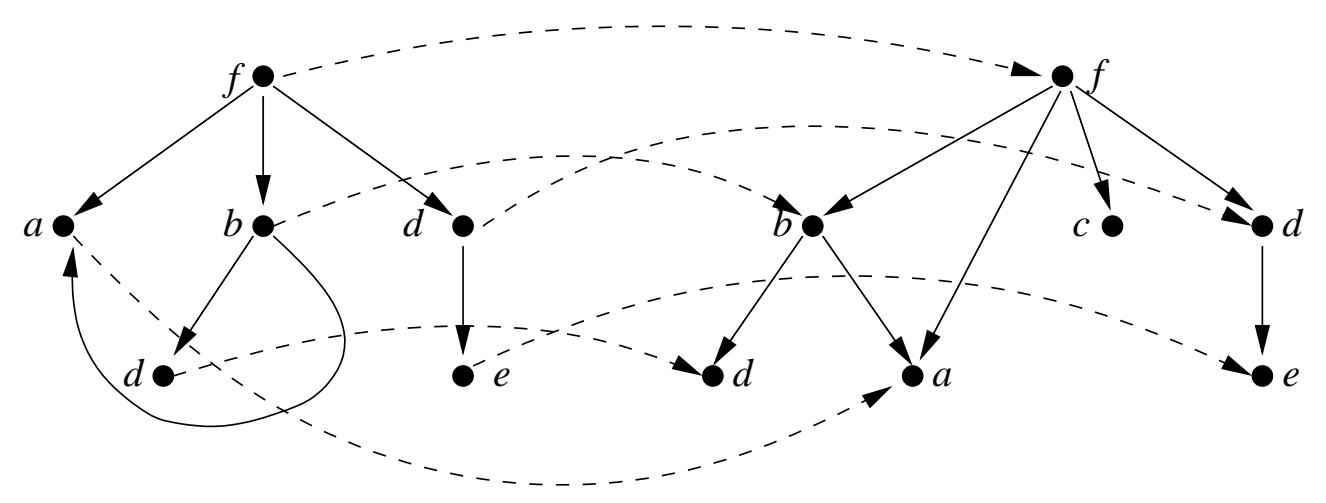

Fig. 2. A simulation of the (graph induced by the) ground query term $t^{q}=$ $f\left\{\left\{\operatorname{id}_{1}: a, b\left[d\{\}, \uparrow \mathrm{id}_{1}\right]\right.\right.$, desc $\left.\left.e\right\}\right\}$ in the (graph induced by the) database term $t^{d b}=f\left[b\left[d, \operatorname{id}_{2}: a\right], \uparrow \operatorname{id}_{2}, c, d\{e\}\right]$.

An answer in a database $D$ to a query term $t^{q}$ is characterised by bindings for the variables in $t^{q}$ such that the database term $t$ resulting from applying these bindings to $t^{q}$ is simulated in an element of $D$. Consider e.g. the query $t^{q}=f\{\{X \leadsto g\{\{b\}\}, X \leadsto g\{\{c\}\}\}\}$ against the database $D=$ $\{f\{g\{a, b, c\}, g\{a, b, c\}, h\}, f\{g\{b\}, g\{c\}\}\}$. The $\leadsto$ constructs in $t^{q}$ yields the constraint $g\{\{b\}\} \preceq X \wedge g\{\{c\}\} \preceq X$. Matching $t^{q}$ with the first database term in $D$ yields the constraint $X \preceq g\{a, b, c\}$. Matching $t^{q}$ with the second database term in $D$ yields the constraint $X \preceq g\{b\} \wedge X \preceq g\{c\} . g\{b\} \preceq X \wedge g\{c\} \preceq X$ is not compatible with $X \preceq g\{b\} \wedge X \preceq g\{c\}$. Thus, the only possible value for $X$ is $g\{a, b, c\}$, i.e. the only possible answer to $t^{q}$ in $D$ is $f\{g\{a, b, c\}, g\{a, b, c\}, h\}$.

Definition 10 (Ground Instances of Query Terms). A grounding substitution is a function which assigns a label to each label variable and a database term to each term variable of a finite set of (label or term) variables. Let $t^{q}$ be a query term, $V_{1}, \ldots, V_{n}$ be the (label or term) variables occurring in $t^{q}$ and $\sigma$ be a grounding substitution assigning $v_{i}$ to $V_{i}$. The ground instance $t^{q} \sigma$ of $t^{q}$ with respect to $\sigma$ is the ground query term that can be constructed from $t^{q}$ as follows:

1. Replace each subterm $X \leadsto t$ by $X$.

2. Replace each occurrence of $V_{i}$ by $v_{i}(1 \leq i \leq n)$.

Requiring in Definition 2 desc to occur to the right of $\leadsto$ makes it possible to characterise a ground instance of a query term by a grounding substitution. This is helpful for formalising answers but not necessary for language implementions. Not all ground instances of a query term are acceptable answers, for some instances might violate the conditions expressed by the $\leadsto$ and desc constructs.

Definition 11 (Allowed Instances). The constraint induced by a query term $t^{q}$ and $a$ substitution $\sigma$ is the conjunction of all inequalities $t \sigma \preceq X \sigma$ such that $X \leadsto t$ is a subterm of $t^{q}$ not of the form desc $t_{0}$, and of all expressions $X \sigma \triangleleft t \sigma$ (read " $\sigma \sigma$ subterm of $X \sigma$ ") such that $X \leadsto$ desc $t$ is a subterm of $t^{q}$, if $t^{q}$ has such subterms. If $t^{q}$ has no such subterms, the constraint induced $t^{q}$ and $\sigma$ is the formula true. Let $\sigma$ be a grounding substitution and $t^{q} \sigma$ a ground instance of $t^{q} . t^{q} \sigma$ is allowed if: 
1. Each inequality $t_{1} \preceq t_{2}$ in the constraint induced by $t^{q}$ and $\sigma$ is satisfied.

2. For each $t_{1} \triangleleft t_{2}$ in the constraint induced by $t^{q}$ and $\sigma, t_{2}$ is simulated in a subterm of $t_{1}$.

Definition 12 (Answers). Let $t^{q}$ be a query term and $D$ a database. An answer to $t^{q}$ in $D$ is a database term $t^{d b} \in D$ such that there exists an allowed ground instance $t$ of $t^{q}$ satisfying $t \preceq t^{d b}$.

\section{Conclusion}

This article introduces the rule-based XML query and transformation language Xcerpt. While the World Wide Web Consortium [6] has proposed XQuery as a generic XML query language, rule-based querying may be advantageous in cases involving more complex queries. Rule-based querying arguably allows for programs that are easier to grasp because of a clearer separation of construction and query parts.

In [11], a more detailed presentation of simulation unification is given and a prototype is currently being worked on [19].

\section{References}

1. W3C http://www.w3.org/TR/xquery/: XQuery: A Query Language for XML. (2001)

2. Fernandez, M., Siméon, J., Wadler, P.: XML Query Languages: Experiences and Examplars. Communication to the XML Query W3C Working Group (1999)

3. W3C http://www.w3.org/Style/XSL/: Extensible Stylesheet Language (XSL). (2000)

4. Buneman, P., Fernandez, M., Suciu, D.: UnQL: A Query Language and Algebra for Semistructured Data Based on Structural Recursion. VLDB Journal 9 (2000) 76-110

5. Henzinger, M.R., Henzinger, T.A., Kopke, P.W.: Computing Simulations on Finite and Infinite Graphs. Technical report, Computer Science Department, Cornell University (1996)

6. World Wide Web Consortium (W3C) http://www.w3.org/. (2002)

7. Chamberlin, D., Fankhauser, P., Marchiori, M., Robie, J.: XML query use cases. W3C Working Draft 20 (2001)

8. Olteanu, D., Meuss, H., Furche, T., Bry, F.: XPath: Looking Forward. In: Proceedings of Workshop on XML Data Management (XMLDM), http://www.pms.informatik.unimuenchen.de/publikationen/\#PMS-FB-2002-4, Springer-Verlag LNCS (2002)

9. Maier, D.: Database Desiderata for an XML Query Language. In: Proceedings of QL'98 - The Query Languages Workshop. (1998) http://www.w3.org/TandS/QL/QL98/.

10. Abiteboul, S., Buneman, P., Suciu, D.: Data on the Web. From Relations to Semistructured Data and XML. Morgan Kaufmann (2000)

11. Bry, F., Schaffert, S.: Towards a Declarative Query and Transformation Language for XML and Semistructured Data: Simulation Unification. In: Proceedings of the Int. Conf. on Logic Programming (ICLP), Copenhagen, Springer-Verlag LNCS (2002)

12. W3C http://www.w3.org/TR/xhtml1/: XHTML 1.0: The Extensible HyperText Markup Language. (2000)

13. WAP Forum http://www.wapforum.org: Wireless Markup Language (WML). (2000)

14. W3C http://www.w3.org/TR/xmlschema-0/: XML Schema Part 0: Primer. (2001)

15. W3C http://www.w3.org/TR/xmlschema-1/: XML Schema Part 1: Structures. (2001)

16. W3C http://www.w3.org/TR/xmlschema-2/: XML Schema Part 2: Datatypes. (2001)

17. Wall, L., et al: Practical Extraction and Report Language, http://www.perl.com/. (19872002)

18. Lucent Technologies, Bell Labs http://cm.bell-labs.com/cm/cs/what/smlnj/: Standard ML of New Jersey. (1996)

19. Schaffert, S.: Xcerpt Prototype, http://demo.xcerpt.org. (2002) 